\title{
Interférences
}

Ars scribendi

$7 \mid 2014$

Le savoir sur la langue

\section{Hermogenes Kratylos: un tentativo di ricostruzione del loro pensiero}

Cratyle et Hermogène: une tentative de reconstruction de leur pensée

\section{Fabio Stella}

\section{(2) OpenEdition \\ Journals}

Édition électronique

URL : http://journals.openedition.org/interferences/4677

DOI : 10.4000/interferences.4677

ISSN : $1777-5485$

Éditeur

HiSoMA - Histoire et sources des Mondes antiques

Référence électronique

Fabio Stella, «Hermogenes Kratylos: un tentativo di ricostruzione del loro pensiero », Interférences [En ligne], 7 | 2014, mis en ligne le 11 décembre 2014, consulté le 15 septembre 2020. URL : http:// journals.openedition.org/interferences/4677 ; DOI : https://doi.org/10.4000/interferences.4677

Ce document a été généré automatiquement le 15 septembre 2020

Tous droits réservés 


\title{
Hermogenes Kratylos: un tentativo di ricostruzione del loro pensiero
}

\author{
Cratyle et Hermogène: une tentative de reconstruction de leur pensée
}

\author{
Fabio Stella
}

1 Facendo seguito alla nota presentata lo scorso Dicembre a Parigi, nella quale, in via del tutto preliminare, delimitavo l'ambito del mio possibile contributo al nostro tema, il Cratilo di Platone, nel senso di una possibile indagine delle "concezioni" del linguaggio, più o meno strutturate, pregresse e/o sottese al dialogo stesso (alla luce della diffusione della scrittura tra l'età greca arcaica e l'età classica e delle "grammatizzazioni" da questa innescate), desidero oggi concentrare l'attenzione sulle due figure di Cratilo, appunto, e di Ermogene, protagonisti con Socrate del dialogo dedicato al primo dei due.

2 Se il titolo di questa comunicazione fa cenno alla "ricostruzione" del loro pensiero, l'indicazione è da intendersi, dunque, nel senso di un tentativo di cogliere il contributo rispettivo che entrambi, ammesso ve ne sia, avessero eventualmente portato nella determinazione dell'humus sulla quale si innesta la riflessione platonica sul linguaggio. Il che da un lato significa, inevitabilmente, esulare dal contesto di una riflessione strettamente linguistica, cui neppure il Cratilo può essere facilmente circoscritto (implicando lo stretto rapporto con i concetti di verità e di essere); dall'altro non si promette neppure, con altrettanta chiarezza, una sistematica disamina di tutti $\mathrm{i}$ possibili riferimenti che la tradizione, come le interpretazioni recenti, hanno creduto di riportare ai personaggi Cratilo ed Ermogene.

Il campo d'indagine è, nondimeno, scivoloso e costringe per sua natura ad un procedimento di tipo indiziario, che conduce a formulare dubbi più che certezze ed impone la massima cautela. Le considerazioni che seguono avranno dunque sempre il carattere dell'ipotesi di lavoro, corroborata sì da echi e riscontri, ma pur sempre "in discussione". Valga questa avvertenza iniziale per tutto quel che segue, anche quando, per esigenze espositive, questa condizione non venisse esplicitamente richiamata.

Occorre non di meno avvertire, infine, che spesso farò riferimento alle mie convinzioni in merito ai "sapienti" arcaici (continuo ad usare per essi la denominazione di Giorgio 
Colli) e alla natura non filosofica dei loro scritti. Non essendo tale questione oggetto dell'odierna riflessione, non potrò soffermarmi sulle argomentazioni, del tutto eterodosse, peraltro, che mi inducono a privilegiare tale prospettiva; mi permetto di rimandare, per questo, a quanto da me esposto a Dicembre in merito al rapporto tra scrittura greca e nascita del pensiero astratto (e, in particolare, alla mia supposizione di una "grammatizzazione" magnogreca - con Teagene e Parmenide - altra ancora da quella pitagorica), oltreché, ovviamente, al dibattito che oggi eventualmente seguisse.

Il presente breve lavoro, in definitiva, vorrebbe presentarsi come una tappa intermedia, del tutto provvisoria, in vista dell'elaborazione dell'ulteriore ricerca sul tema del nostro progetto.

Il mio discorso ruoterà attorno a tre questioni principali, di tipo contenutistico, ed una domanda, propedeutica alle altre, di natura storica.

Risulta infatti doveroso porre preliminarmente la domanda intorno all'esistenza reale di Ermogene e di Cratilo, alle determinazioni geografiche e temporali del loro agire, in particolare rispetto a Socrate prima e a Platone poi. Quindi potranno seguire gli interrogativi più sostanziali:

a. quello circa il rapporto tra i due pensatori e il presunto "eleatismo" dell'uno ed "eraclitismo" dell'altro (il che comporterà la tematizzazione, per quanto scabrosa, dei possibili riflessi del Parmenide e dell'Eraclito storici entro il loro eclettismo);

b. quello in merito all'importanza maggiore, così in genere si è interpretato, di Cratilo rispetto ad Ermogene (che si vorrebbe, fin nella "finzione" del dialogo, relegato dal Socrate platonico - cioè da Platone medesimo - al ruolo di "diligente scolaro" e, in assoluto, provvisto di un pensiero meno robusto, inficiato, appunto, da una volenterosa quanto confusa eterogeneità;

c. quello, conclusivo, attorno al ruolo che le posizioni dei due amici di Platone possono aver svolto rispetto all'elaborazione del Cratilo, nell'evoluzione della filosofia platonica, nella determinazione della lettura tradizionale del pensiero cosiddetto "pre-socratico".

Le questioni, come è evidente, si implicano vicendevolmente, ma cercherò di tenerle, per esigenze di chiarezza espositiva, il più possibile distinte.

Procediamo, dunque, con ordine.

\section{Premessa}

10 Di Cratilo si è messa in discussione persino l'esistenza e, quanto all'identificazione, non sono mancate le tesi più varie: quella, di vecchio conio, che lo vorrebbe maschera di Eraclide Pontico, scolaro di Platone; quelle, anche relativamente recenti, che lo vorrebbero prestanome di un sofista: di Antistene o di Protagora (è il caso quest'ultimo, tra gli altri, di Michel Narcy nel suo «Cratyle par lui-même» - per quanto egli parli più precisamente di "proximité et identification éventuelle") o di Antistene (ad es. Manlio Buccellato).

11 Personalmente sono portato a ritenere, da un lato, che l'eventuale prossimità di Cratilo ad uno dei grandi retori è plausibile ma dice solo che il clima culturale tra v e IV secolo a.C. era condizionato, se non determinato, dalle idee della Sofistica e non che dietro il nome Cratilo si nasconda un personaggio in particolare; dall'altro, penso che occorra non dimenticare che il Cratilo di cui leggiamo le peraltro scarne risposte a Socrate, quello sì che è figura letteraria creata da Platone, forse anche fededegna, ma 
certo non costruita con l'intento di testimoniare il vero storico. E poi c'è Aristotele, che, anche qualora non si voglia dar piena fiducia agli altri, e pur essendo egli parimenti animato da uno spirito teoretico e non da storico, difficilmente sarebbe giunto alla replica della supposta falsificazione platonica, o comunque occorrerebbe trovarne le ragioni.

Ritengo al contrario di altri possibile ma, a ben vedere, non essenziale, la tesi, infine, di Anastase N. Zoumpos, che lo vorrebbe identificato con quel Crate o Cratete, che avrebbe rubato il libro di Eraclito dal tempio di Artemide ad Efeso. Cratilo si atteggiava a conoscitore di Eraclito: che il libro lo avesse portato lui ad Atene o vi fosse giunto per altra via (magari per il tramite di qualche discepolo dell'altro Antistene, l'eracliteo efesio, o ancora diversamente) probabilmente poco importa, stante che dell'Eraclito storico ben poco veniva compreso.

Platone ci dice, nel dialogo a lui dedicato, che Cratilo era figlio di Smicrios e ateniese, per quanto lo affermi in un contesto particolare (Socrate dialoga con Cratilo e gli presenta l'eventualità che egli sia appellato in un paese straniero col nome di Ermogene) e attorno al patronimico si sia pure discusso, sospettandolo un epiteto ironico.

Gli interpreti più recenti sono invece piuttosto concordi nel porre la data di nascita di Cratilo attorno al 450. Peraltro Platone, sempre nel Cratilo, ci dice che egli era ancor giovane quando Callia minor aveva già speso molti denari presso Protagora e i Sofisti, ovvero poco dopo esser venuto in possesso nel 425 delle sostanze familiari accumulate dal vecchio Callia, chiamato lakkòploutos "ricchezza di buco", perché arricchitosi con le miniere del Laurion. Egli era dunque di una ventina d'anni più giovane di Socrate e di altrettanti anni più vecchio di Platone.

Aristotele ci dice infatti che fu maestro del giovane Platone, cui trasmise le dottrine eraclitee. Se questo è vero, se ne deve dedurre che Platone ebbe a che fare con Cratilo in almeno due occasioni diverse: in qualità di discepolo prima, ritrovandolo poi, da adulto, dopo la morte di Socrate, come ci conferma Diogene Laerzio.

Della storicità di Ermogene è impossibile dubitare. Platone e Senofonte ce ne parlano, lo conobbero e lo frequentarono. Egli era il figlio minore di Ipponico, il figlio del medesimo Callia lakkòploutos di cui dicevamo a proposito di Cratilo e dunque fratello di Callia minore. Egli non ereditò però il patrimonio di famiglia, facendo una vita decisamente più sobria, ed è ricordato da Senofonte per la scarsa propensione alle ricchezze, per la generosità e religiosità, per la sesnsibiltà musicale. Se il fratello voleva pagarsi la scuola dei sofisti, egli frequentava Socrate fin da giovane, assistette al processo del 399 e fu con lui nel momento della morte. Subito dopo, forse, come molti dei discepoli più prossimi al maestro, si rifugiò a Megara, presso Euclide, per timore di ulteriori rappresaglie, rimanendo poi lì alcuni anni, visto che ritroviamo la sua presenza ad Atene attestata solo nel 392, quando fece parte di una delegazione presso il persiano Tiribazo.

17 Platone lo conobbe verosimilmente, dunque, all'interno del circolo socratico, quando si accostò al grande ateniese dopo la giovanile conoscenza di Cratilo; lo perse di vista, come pure fece con l'“eracliteo", durante i suoi viaggi fuori da Atene; ritrovò entrambi dopo la fondazione dell'Accademia.

Antonio Capizzi, uno dei pochissimi studiosi ad aver dedicato ad Ermogene un saggio, ipotizzava per le diverse fasi, non senza ragioni che personalmente condivido, le date 
seguenti: riportava al 409-406 il primo incontro e al 366-364 (la collocazione tarda implica, come è evidente, una datazione altrettanto del dialogo, per nulla affatto condivisa per quanto tra le possibili) il nuovo sodalizio, al culmine di percorsi autonomi, diversi per quanto, infine, "convergenti". Quasi unanimemente il Cratilo è collocato, infatti, nella fase della "maturità" di Platone, tra la stesura del Simposio e quella del Teeteto.

Non ho volontariamente fatto cenno al presunto "eleatismo" (testimoniato dalla notizia di Diogene Laerzio, secondo cui Ermogene sarebbe stato, nell'interpretazione di Parmenide, l'omologo di Cratilo esegeta di Eraclito - il suo avversario "moderno", secondo la visione tradizionale codificata che vuole opposti i due sapienti arcaici), perché trattasi del tema principale affrontato nell'analisi che segue.

\section{Eraclitei ed Eleatici in Atene}

Cominciamo anzitutto col dire che ritengo un eventuale giudizio che voglia le riflessione di Cratilo più vicina, per quanto frutto di interpretazione, al pensiero di Eraclito rispetto a quanto la posizione di Ermogene sia riportabile (tengo per un attimo per buona la testimonianza di Diogene Laerzio), a Parmenide, un esercizio privo di fondamento. Poco o nulla tra v e IV secolo ad Atene si poteva comprendere di testi (uno peraltro poetico e fortemente allusivo), elaborati in luoghi distanti e in contesti storici, diversi tra di loro oltre che lontani ormai almeno settant'anni da quelli dei loro esegeti. La presunta asimmetria poggia sul fatto che il "panta rei" cratileo avrebbe un qualche rapporto con la "dottrina degli opposti" di Eraclito, mentre la concezione nominalistica del linguaggio di Ermogene sarebbe molto più a fatica riportabile al frammento 8 , versi 53-61 di Parmenide che alle concezioni del linguaggio proprie della Sofistica o del contemporaneo Democrito.

21 In realtà, i rapporti con i due "sapienti" antichi appaiono parimenti labili in entrambi $i$ casi e dunque, paradossalmente, parimenti plausibili, qualora per "rapporto" si intenda un'arbitraria interpretazione di lacerti di discorso, senza la reale comprensione, pur a fronte della lettura integrale dei testi, del significato d'insieme.

Ipotizzo, insomma, che sia Cratilo che Ermogene partano da posizioni relative al linguaggio (la "naturalistica" e la "convenzionalistica", per semplificare) desunte dal dibattito loro contemporaneo, e che si siano poi rivolti ai testi arcaici, cercando di piegarne, isolandoli, alcuni passaggi alle rispettive posizioni, nel tentativo di puntellarle.

In questo non erano i soli, visto che la testimonianza platonica nel Teeteto fa riferimento ad interi gruppi, peraltro oscuri, i "parmenidei" e gli "eraclitei", dediti all'improbabile esegesi (Tht. 179d-181b), tra cui occorre scegliere e parteggiare.

Lo stesso Cratilo sembra ammettesse che del libro di Eraclito (da lui stesso portato ad Atene, come dicevamo, o già presente ad Atene per altra via) egli, nonostante gli sforzi, ci avesse capito poco o niente (la notizia ce la riporta Seleuco) e, come è noto, Aristotele ci riferisce nella Metafisica (IV, 5, 1010a) dell'estremizzazione che egli avrebbe operato rispetto ai frammenti sui fiumi: neppure la prima ed unica, e non già una seconda volta, non si potrebbe scendere nello stesso fiume, visto il mutare incessante dello stesso. 
quali il nome è sempre corretto perché legato naturalmente alla cosa nominata (complicato dalla parallela accettazione dell'impossibilità di dire il falso, visto che si emettebbe un semplice suono senza riferimento alcuno, e da quello che sembra essere l'esito finale delle premesse (sempre Aristotele in Metaph. IV, 5, 1010a): la realtà non può essere descritta con i nomi, solo indicata con i gesti - "mutismo" di Cratilo). Che "l'impossibilità di dire il falso» sia corollario alla tesi "naturalistica" iniziale è plausibile, per quanto non si configuri esattamente come una conseguenza; che l'impossibilità del parlare e il «doversi limitare a muovere semplicemente il dito» siano asserzioni conciliabili a loro volta col "naturalismo" cratileo, in grazia della natura "ostensiva" del nome (come, tra gli altri suppone Francesco Aronadio) comporterebbe almeno un passaggio intermedio, che istituisse, ad esempio, un gradiente o un'alternativa; resterebbe ad ogni modo fuori, come difficilmente accordabile, la "radicalizzazione" dei frammenti sui fiumi.

Concentriamo l'attenzione su quest'ultimo punto.

In nome del presunto eraclitismo, dato per certo, che sarebbe riflesso nella tesi dello scorrere della realtà, ci si domanda, in genere, se anche le posizioni sul linguaggio non abbiano in qualche modo una matrice eraclitea, senza che questa sia in verità rintracciabile, se non a costo di acrobazie interpretative, non autorizzate alla luce dei frammenti a noi giunti (meno che mai dal frammento $1 \mathrm{DK}$, neppure nella sua lettura più tradizionale, in quanto, ammettendo - e non lo credo - che il logos ivi sia detto "conforme alla natura delle cose", questo è, come è stato notato, «ben diverso dal dire che esiste per natura un modo corretto di nominare le $\left.\operatorname{cose}^{1}{ }^{1}\right)$.

Ritengo che la domanda vada diametralmente rovesciata: stante che Cratilo assume la posizione sofistica suddetta (verosimilmente protagorea o antistenica, o ancora variatamente eclettica) relativamente all'orthótes dei nomi, come giunge, nel tentativo di difenderla o di risolverne le aporie interne, a leggere nei frammenti dei fiumi un Eraclito inesistente fin nelle premesse o comunque misinterpretato al limite della falsificazione?

La risposta l'ha recentemente fornita Pierre Ponchon, nel suo recente lavoro dal titolo " Cratyle et la réception d'Héraclite à Athènes»; ${ }^{2}$ o meglio, è possibile ricavarla dalla sua analisi, a patto che la si emendi della visione, abbastanza classica, del testo di Eraclito e dall'idea che Cratilo parta di lì.

30 Egli ritiene infatti, a partire da un passo del Cratilo, siamo nella lunga e problematica parte centrale sulle etimologie, ove Socrate presenta la doppia radice del nome di Zeus (Cra. $396 \mathrm{a}-\mathrm{b})$, traduce il frammento 32 DK di Eraclito, che ha un semplice valore etico «C'è un'unica cosa saggia: pronunciare, lo si voglia o meno, il nome di Zeus» - con il classico «L'Un, la chose sage, il veut et ne veut pas que le nom de Zeus lui soit donné seul», in modo da fare del passo citato una possibile risposta di Cratilo al suo maestro. Parimenti egli fa riferimento al logos (il Socrate platonico dice in effetti che «il nome di Zeus è come un logos») come al discorso (di Eraclito) che si identifica col Discorso (la "dottrina degli opposti", vista pur sempre un principio del fluire), ovvero lo pensa nei termini tradizionali, sostanzialmente metafisici: i nomi, dunque, sono corretti, quando restituiscono la tensione degli opposti entro la loro unità, come indicherebbe la coppia Zéna/Dià ("la vita", che scorre, e "colui che è a capo di tutto", ovvero l'unità).

31 Insomma: il Socrate platonico e il personaggio Cratilo (che, nel suo mutismo, assentirebbe accettando l'etimologia proposta), in un contesto probabilmente ironico 
(Socrate afferma poi che il nome Dià significa che Zeus è progenie di una grande diànoia - intelletto) divengono il criterio per l'interpretazione di Eraclito, laddove, ai tempi di Eraclito, esisteva sì l'equivalenza Zeus = Zàs (la vita), ma in virtù del logos mitico (di una mitizzazione della natura) come quello di Ferecide di Siro (600-596 a.C. - vi sec.).

Pur non volendo escludere nulla (tutti disegnano trame meramente indiziarie) mi pare più produttivo, come dicevo, ipotizzare un procedere inverso: le tesi di Cratilo sui nomi sono quelle che, nell'Atene del suo tempo, si discutevano all'interno del più ampio dibattito su "natura e convenzione", la sua passione (in fieri, come testimoniato dal suo impegno a meditare con cui si chiude il Cratilo) per il libro di Eraclito mi appare un tentativo di utilizzarne i presunti argomenti arcaici entro quel dibattito.

Che dietro a Cratilo si "nasconda", dunque, questo o quel sofista, o l'autore del Papiro di Derveni, come leggeva Walter Burkert (Anassagora?), o Eraclide Pontico o altri ancora, non dice di necessità che egli sia un prestanome di qualcuno in particolare: fu un eclettico, al pari di Ermogene e di tanti contemporanei, che Platone nel Cratilo mostra di rispettare, quanto di utilizzare per criticare l'intera Sofistica e, dal suo punto prospettico, la mancanza di fondamento che la caratterizza, pur nell'apprezzamento di alcuni aspetti e nel rispetto degli esponenti maggiori, in generale e relativamente al linguaggio, abbozzando infine, grazie a tale lavorìo, la sua "terza via" tra convenzione e natura.

Per il resto, il saggio di Ponchon, cui rimando senz'altro, offre una ricostruzione convincente dei possibili fasi dell'elaborazione dell' "eraclitismo" cratileo.

Un primo passaggio sarebbe, appunto, caratterizzato dall'influenza di Protagora nella posizione del problema dell'orthoépeia (la "corretta elocuzione") e avrebbe come momento decisivo l'operazione (dedotta dal Teeteto, 157b, dove la si attribuisce agli "eraclitei"), di sostituzione dei nomi propri, dei nomi comuni e dei pronomi con il participio presente dei verbi, alla ricerca di una congruenza tra flusso della realtà e fluire del discorso, aprendo ad «une approche de plus en plus "performative" du langage ${ }^{3} »$ (p. 57). Resterebbe da chiarire se in questo momento il reale come "flusso" sia concetto ancora di matrice protagorea (penso alla "materia scorrevole" propria dell' héttôn logos di Protagora nella nota interpretazione di Mario Untersteiner, che, infatti, la riportava piuttosto a Pitagora), come mi appare probabile, o già frutto, in aggiunta, di una qualche contaminazione con Eraclito.

Un successivo momento vedrebbe la tematizzazione della discrasia tra tempo del fluire delle cose e tempo del discorso, di cui sia avrebbe eco nel Cratilo (439d-e), e che, in una prima radicalizzazione della dottrina del flusso, porterebbe all'impossibilità del parlare stesso, in quanto, per così dire, la realtà è sempre più velocemente mutevole del linguaggio che tenta di dirla (in qualche modo, di afferrarla).

La somiglianza che si potrebbe istituire dell'argomento con i paradossi zenoniani, in particolare con quello di "Achille e la tartaruga", testimonierebbe, infine, il ruolo dell' hetàiros di Parmenide nella genesi dell'"eraclitismo" cratileo più estremo (cosa che appunto la visione classica delle due scuole contrapposte rifiuterebbe a priori), causando la seconda, più famosa, radicalizzazione della teoria del flusso, quella dei frammenti sui fiumi; ovvero facendo sì che un problema di velocità relativa divenisse un problema di differenza "qualitativa" tra i due flussi: la divisibilità finita di uno spazio finito contro le unità discrete rappresentate dai suoni, non infinitamente divisibili (ricordo che, all'inizio del Cratilo, i due interlocutori sembrano venire da una discussione pregressa, che Raffaella Petrilli $^{4}$ ha ipotizzato vertesse su una 
"grammatizzazione" di origine pitagorica). Dunque «non si può scendere neppure una volta nello stesso fiume» e, stante il fluire delle cose, non resta che rinunciare al linguaggio e alla pretesa di designare le cose, in favore del designare, con i gesti, il flusso universale altrimenti indicibile.

Interessante davvero, ai miei occhi, questo Cratilo che deve all'"eleatismo" (del v secolo ateniese) non meno che quell'eraclitismo, che in gran parte instaura. Non ci si scandalizzerà più di tanto se Ermogene dovesse risultare altrettanto eclettico e figura non meno importante.

\section{Ermogene: un interlocutore minore?}

Dell'eleatismo di Ermogene si dubita in genere di più rispetto a quanto lo si faccia dell'eraclitismo del suo interlocutore, e non a torto. Oggi si è portati a svalutare le parole di Diogene Laerzio, in quanto sarebbero viziate proprio dalla volontà di opporre, schierando su posizioni avverse i tardi discepoli, i due sapienti arcaici, secondo schema divenuto poi classico. Nel Cratilo, in effetti, Parmenide non viene mai citato, mentre Eraclito esplicitamente sì. D'altro canto quasi tutti gli interpreti, pur dubbiosi, hanno considerato la posizione "convenzionalista" di Ermogene in relazione alla correttezza dei nomi "accordabile", in senso lato, con il frammento 8 del poema parmenideo, laddove, ai versi 53-61, si parla (non mancano certo i problemi interpretativi) dello "stabilimento" dei due segni opposti della Nyx adaès (la Notte oscura o "senza memoria") e dell'aitérion Pyr (Fuoco etereo).

Ora, se il primo indizio non basta ad assicurarci la sua estraneità all'eleatismo, il secondo parimenti non basta ad farne un "parmenideo".

Certo è che Parmenide inizia a diventare un riferimento importante per Platone, e sempre di più lo sarà, a partire dal Teeteto. Ma donde proviene a Platone la conoscenza delle dottrine, vere o presunte, del "sapiente" di Elea?

Il poema di Parmenide era in Atene, portatovi da Zenone, fin dai tempi di Pericle, ma Platone non mostra di averne fatto uso (o di presentarne l'autore) prima di una certa data, quella di stesura del Teeteto (o del Parmenide, a seconda dell'antecedenza accertata dell'una o dell'altra opera). Non è da supporsi, dunque, una conoscenza, per quanto mediata, scaturita dai suoi viaggi in Magna Grecia (da cui, piuttosto, derivano il Pitagorismo e gli influssi empedoclei): al ritorno dai viaggi, egli ne avrebbe dovuto mostrare, verosimilmente, una qualche eco.

Ma neppure è ipotizzabile, come Capizzi ritenne, mimando, nella sostanza, la visione "classica" in merito, una derivazione di questa dalla scuola megarica, visto che, come ha dimostrato in modo pressoché conclusivo Gabriele Giannantoni, ${ }^{5}$ la presunta "contaminazione" tra socratismo ed "eleatismo" non è mai avvenuta ed è frutto di interpretazioni e distorsioni posteriori (come già supposto da Kurt von Fritz).

Resta vero, però, che Ermogene rimase a Megara tanto da poter recepire la dottrina della scuola e che poi lo ritroviamo nell'Accademia con Platone. Il che, se si deve escludere, per i motivi suddetti, che egli sia il tramite di un improbabile "eleatismo megarico", lascia aperta la strada alla possibilità di una qualche influenza delle lezioni di Euclide sulla sua formazione e sugli sviluppi successivi del suo pensiero.

Continuo, in questa direzione, a ritenere che la misinterpretazione del tò eón parmenideo che Platone presenta nel Teeteto, possa rivelarsi un indizio importante per 
comprendere il ruolo di Ermogene. Ivi (180e), infatti, il testo del frammento 8, verso 38 (tô pant'ónoma éstai, "perciò saranno tutte nome») diventa tô pantì ónom'eînai («il tutto ha nome»), finendo per attribuire in tal modo al Parmenide platonico quel "il tutto", cui probabilmente il Parmenide storico non aveva mai pensato. Non mi riferisco, sia chiaro, al la mancata presenza del termine pan, che, come semplice aggettivo, è chiaramente riferito all'eón al verso 5 del frammento, ma all'aggettivo sostantivato a mezzo dell'articolo (tò pan).

Che Ermogene sia stato l'autore della distorsione in questione, come voleva Capizzi, resta cioè, ai miei occhi, un'ipotesi interessante, pur a fronte del venir meno dell' "eleatismo megarico" su cui egli, negli anni '80, fondava i suoi ragionamenti; o meglio: proprio in virtù della falsità dell' "eleatismo" megarico, a patto, ovviamente, che io sia in grado di esibire altri elementi a sostegno dell'ipotesi.

Mi limito preliminarmente a notare che, se ciò fosse possibile e la ricostruzione divenisse plausibile, Ermogene ci si presenterebbe, al pari di Cratilo, da un lato come un eclettico esegeta di passi interpretati secondo i propri intenti; dall'altro, come un personaggio tutt'altro che minore, se è vero che orientò o contribuì ad orientare, direttamente o indirettamente, il pensiero di Platone in una direzione così decisiva.

\section{Cratilo ed Ermogene nel dialogo e oltre il dialogo}

48 Chiediamoci, dunque: che rapporti intercorrono, sempre ne esistano, tra la lettura di Parmenide, che supponiamo operata da Ermogene, le tesi sul linguaggio che questi espone nel Cratilo e quelle attribuite ai parmenidei nel Teeteto? A mezzo di quali operazioni esegetiche, e, appunto, compiute da chi, si potrebbero legare, se di legame si tratta, questi passaggi tra loro?

Purtroppo, la natura letteraria della figura di Ermogene così come Platone ce la presenta nei dialoghi (segnatamente nel Cratilo) non permette di isolare con certezza gli elementi della sua dottrina, propria $o$, pur fosse, di matrice megarica, dal contesto; cioè a dire: non è possibile "depurare", ad esempio, le tesi di Ermogene esposte nel Cratilo da quello che il Socrate platonico gli vuol far dire, ed avere ipso facto il pensiero dell'Ermogene storico.

Cionondimeno, lavori come quello di Serafina Rotondaro, ${ }^{6}$ tendenti ad evidenziare il modo con cui Socrate gioca sulle risposte dell'interlocutore, fino a fargli sostenere cose che egli non dice o che dice diversamente, già importanti per la ricostruzione dell'intreccio e degli intenti platonici, se non possono darci certezze, possono però fornire un criterio d'orientamento.

51 È pur vero, infatti, che spesso quel che Platone fa dire ai suoi personaggi è tutto, o quasi, quel che abbiamo. In tal caso, comunemente si ragiona, in mancanza di prove contrarie, come se la testimonianza platonica fosse fededegna; non possiamo, dunque, che ripartire di lì, dalle parole del Cratilo, per, ancora una volta, avanzare delle semplici ipotesi, per quanto non prive d'indizi a sostegno.

52 In particolare, vorrei trascegliere, seguendo la Rotondaro, tra le non molte affermazioni fatte da Ermogene in difesa della tesi per cui la correttezza dei nomi ha come criteri la convenzione e l'accordo (synthéke kai homologhía), la consuetudine e l'uso (nómô kai éthei), quella, messa in evidenza proprio dalla Rotondaro, relativa al "títhesthai " ("porre" il nome) e al "kaléin" ("chiamare" qualcosa con un nome); Ermogene 
acconsente incautamente ad entrambe le proposizioni, in virtù dell'ambiguità del "chiamare" (che può valere tanto "dare un nome" come "utilizzare un nome"); in realtà, egli vuol dire che convenzionale è l'atto nomotetico, ovvero quello del denominare originario, non che ogni "chiamare" sia di volta in volta un atto arbitrario (che nella prassi, al contrario, si deve rispettare la regola comune, pena la non comunicabilità). Una connotazione ben diversa dalla curvatura soggettiva protagorea $\mathrm{e}$ da quella, opposta nella forma, identica negli esiti, di Eutidemo, cui Socrate prontamente invece la riporta, ottenendo così, nonostante Ermogene rifiuti entrambi gli accostamenti, una conseguenza decisiva non voluta dall'interlocutore e non presente nelle sue parole, ovvero che, all'interno di una concezione convenzionalista, di necessità le cose non hanno un'ousía stabile (386a).

53 Da qui gli altri passaggi argomentativi, condotti abilmente da Socrate fino a far dire ad Ermogene che il nome, nei suoni e nelle sillabe, riproduce per imitazione le cose, laddove egli concede solo che, stante un'ousía stabile, i nomi siano posti in modo corretto se riproducono bene l'idea del nome; il che non comporta affatto la contraddizione d'una tesi convenzionalista.

Autenticamente parmenidea è, in un certo senso, la stabilità dell'ousía: il to eón, pur non avendo il carattere metafisico che poi gli si attribuirà, stava a denominare, ritengo, la "cosa esistente" e tutte le cose, in tal senso, "sono", in quanto ciascuna cosa è una "cosa esistente": to eón.?

Se Ermogene, sia detto per assurdo, fosse stato portavoce del falso "eleatismo" megarico, non avrebbe potuto sentire il valore dell'antico to eón parmenideo, perché fin dall'inizio convinto dell'identità tra uno, bene ed essere. Invece lo percepisce, grazie probabilemnte alla conoscenza, già accertata per Cratilo, di Zenone, tramite del poema arcaico.

D'altro canto, autenticamente megarica è la questione "dell'uno e dei molti", del tutto indipendente da ogni eleatismo, ma che potrebbe, così ipotizzo, aver avuto un ruolo per Ermogene, in quanto implicata, probabilmente, nella distorsione del frammento 8, verso 38, di Parmenide (da "le cose sono tutte nome" a "il tutto ha nome"), pur non essendo neanch'essa, al pari dell'eleatismo a qualsivoglia versione ridotto, la causa principale del porsi di tale equivalenza tra pánta "tutte" aggettivo e to pan "il tutto" sostantivo.

57 Cosa poteva spingere, infatti, Ermogene (sempre che di lui si parli) a tale "radicalizzazione", parallela, anche cronologiacamente forse, essendo immaginabile dopo il dialogo intitolato Cratilo, alla "radicalizzazione" operata da Cratilo a proposito dei fiumi?

Il passaggio da "tutte le cose" a "il tutto" non fu, credo (pur non escludendo una sua possibile preparazione, parallela o convergente, all'interno di non meglio precisati circoli "eleatici" del v secolo), né un'eco del presunto "essere" parmenideo, né del "bene" megarico, ma, anzitutto, una necessità interna alle iniziali posizioni ermogenee, di origine sofistica o democritea: se le cose che hanno stabilito i mortali "saranno tutte nome" e la natura convenuta del nome non toglie che ciascuna cosa sia (indipendentemente dall'atto del nominare), questo salva sì il convenzionalismo dal soggettivismo protagoreo (anche qualora Protagora avesse voluto dire solamente, in modo antimetafisico, che la cosa non è conoscibile, se non dal punto prospettico umano) ma non lo salva dalla confutazione del Socrate platonico (di Platone, suo intelocutore reale e quotidiano), che pur sempre vorrebbe non scientificamente 
conoscibile ciò che è plurimo (e, dunque, che il nome che designa ciascun ente dovrebbe ricevere la sua correttezza da qualcosa di ulteriore rispetto alla semplice referenza all'ente stesso).

Di qui la necessità di assegnare il nome al "tutto", alla totalità, ovvero all'"essere", rendendo in tal modo plausibile un convenzionalismo assoluto, essendo ciascun nome sempre e comunque un'atto di denominazione della totalità insieme alla parte.

In questo senso, potrebbe aver avuto un qualche ruolo il ricordo delle posizioni di Euclide in merito alla molteplicità dei nomi del bene (forse uno dei prodromi della successiva mereologia megarica). Se, infatti, le affermazioni riportate da Diogene Laerzio («egli sostenne che il sommo bene era uno, anche se chiamato con molti nomi» e «respingeva tutto quel che fosse contrario al bene, negandone l'esistenza») vanno inquadrate, come ha chiarito Giannantoni, ${ }^{8}$ all'interno di un Socratismo genuino, e «[...] di un intervento diretto nel dibattito apertosi tra i Socratici sull'insegnamento del maestro dopo la sua condanna a morte», è certo che a quel dibattito, e per un tempo più ampio che non Platone, e dunque alla sua piena articolazione, aveva partecipato Ermogene.

61 La trasformazione di Parmenide nel filosofo dell'"essere" si compie, dunque, si può ipotizzare, anche perché Ermogene vuol difendere il convenzionalismo del nome, e l'operazione sarebbe, in questo senso, parte integrante dell'evoluzione del suo pensiero. I due temi, dell'essere e del nome dell'ente, si potrebbe supporre, appaiono strettamente congiunti grazie alle premesse megariche estese indebitamente da Ermogene, in un contesto ateniese e sofistico che dibatteva di natura e convenzione relativamente al linguaggio (o meglio ai nomi e alla loro correttezza), mediante la commistione tra pensiero megarico ed elaborazioni "eleatiche", storicamente mai avvenuta prima (ancora una volta il rimando Giannantoni ${ }^{9}$ e, prima di lui, a Julius Stenzel).

62 In questo senso, debole come si vede, l'eleatismo di Ermogene sarebbe, a patto di riconoscerne la natura, comprovato, e la testimonianza di Diogene Laerzio su di lui, opportunamente filtrata, non del tutto fuorviante; anzi, per altro verso la si potrebbe persino definire "pienamente attendibile", se l'eleatismo fosse ciò che Ermogene contribuì verosimilmente a teorizzare, ovvero se l'eleatismo prima di lui fosse stato mai basato sull'identificazione tra "il tutto" e l'"essere". Cosa che, almeno questa, semplicemente non fu (neppure in Melisso c'è alcuna traccia de "il tutto"; anch'egli subisce la stessa sorte di Parmenide, per mano a sua volta, sembrerebbe, di Polibo, successore di Ippocrate alla Scuola medica o delle sue fonti ${ }^{10}$ ).

Platone potrà, tra la maturità e la vecchiaia, recepire i risultati delle due "radicalizzazioni" in atto nei termini consoni alla sue teoresi e farne rispettivamente la tesi sostenute dai "parmenidei immobilizzatori - stasiótai" e dagli "eraclitei scorrenti rhéontes", iniziando così, grazie ad Ermogene, la sua ghigantomachía perì tês ousías, il suo confronto col venerando sapiente di Elea.

64 Mi guardo bene, a questo punto, avendo probabilmente già fatto abuso di ragionamenti ipotetici, di addentrarmi nei meandri del Teeteto, tra la cornice "megarica", l'assenza delle Idee o la centralità (nella seconda parte, frutto, con ogni probabilità, del rifacimento tardo) del tema dell'essere e della differenza. Troppe le suggestioni, cui farebbero da contraltare troppe difficoltà interpretative, impossibili da districare in questa sede. 


\section{BIBLIOGRAPHIE}

\section{Textes anciens}

Platone, Cratilo, trad. e introd. di Fr. Aronadio, Roma - Bari, 1996.

Platon, Cratyle, trad. inédite, introd., notes, bibliographie et index par C. Dalimier, G.F. 954, Paris, 1998.

Platone, Teeteto, a cura di Fr. Ferrari, Milano, 2011.

\section{Textes modernes}

ADEMOLLO Fr. 2011, The Cratylus of Plato. A Commentary, Cambridge.

ARONADIO F. 2011, I fondamenti della riflessione di Platone sul linguaggio. Il Cratilo, Pleiadi 14, Roma.

CAPIzZI A. 1995, «Ermogene, figlio di Ipponico», in A. Capizzi, Paradigma, mito, scienza. Studi sul

pensiero greco, Filologia e critica 75, pp. 365-371.

Colli G. 1981, La nascita della filosofia, 4a ed., Piccola biblioteca Adelphi 29, Milano.

GIANNANTONI G. 1990, Socratis et socraticorum reliquiae, Elenchos 18, Napoli, 4 voll.

NARCY M. 1987, «Cratyle par lui-même», RPhA 5, pp. 151-165.

PETRILli R. 2009, Linguaggio e filosofia nella Grecia antica. Tra i Pitagorici e Aristotele, Temi e Testi.

Tribunali della fede 70, Roma.

PONCHON P. 2011, «Cratyle et la reception d'Héraclite à Athènes», Méthexis 24, pp. 45-66.

Interférences, 7 | 2014 
ROTONDARO S. 2005, «In difesa di Ermogene», in G. Casertano (a cura di), Il Cratilo di Platone.

Struttura e problematiche, Skepsis 18, Napoli, pp. 42-51.

STELLA F. 2007, «Scrittura greca e nascita del pensiero astratto», in G. Coccoli (a cura di), La mente, il corpo e i loro enigmi. Saggi di filosofia, Filosofía 2, Roma, pp. 247-265.

UNTERSTEINER M. 1996, I sofisti, Sintesi, Milano.

\section{NOTES}

1. Dalimier (a cura di) 1998.

2. Ponchon 2011.

3. Ibid., p. 57.

4. Petrilli 2009.

5. Giannantoni 1990, IV, pp. 51-60.

6. Rotondaro 2005.

7. Sono convinto che il significato dell'eón parmenideo derivi dall'analisi metalinguistica, inaugurata da Teagene e resa possibile dalla scrittura, del testo omerico, tesa alla scomposizione degli elementi costitutivi del discorso: il participio del verbo essere, fuori dal continuum fonico della recitazione orale, venne da Parmenide isolato come "nome grammaticale", in quanto "base comune" per ciò che indica, di volta in volta, l'aggettivo ad essa congiunto; così, ad es., in Il. V,

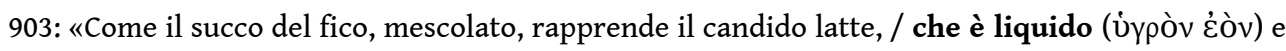
a un tratto s'addensa, mentre si gira, / così di colpo egli guarì Ares ardente».

8. Giannantoni 1990, pp. 58-59.

9. Ibid., nota 6, pp. 61-71.

10. Capizzi 1995, p. 368.

\section{RÉSUMÉS}

Il faut comprendre la « reconstruction » de la pensée de Cratyle et Hermogène dans le sens d'une tentative de comprendre leur contribution respective à la constitution d'un terrain sur lequel se construit la réflexion platonicienne sur la langue. D'une part, cela signifie, inévitablement, sortir du cadre d'une réflexion strictement linguistique, sans d'autre part prétendre à un réexamen complet de toutes les références possibles que la tradition a cru bon de relier aux deux personnages de Cratyle et Hermogène.

fiction du dialogue, héraclitisme, imitation, linguistique, philosophes éléates, philosophie mégarique

fiction in dialogue, Heraclitism, imitation, linguistics, Eleatic school, Megaric philosophy

Rebuilding Cratylus' and Hermogenes' thought must be intended here as an attempt to identify the common basis upon which Plato has built his own thought on language. It means that this paper necessarily goes out of the strict field of linguistics but does not aim to take into account the whole preserved tradition on Cratylus and Hermogenes. 
INDEX

nomsmotscles Antisthène, Aristote, Artémidore d'Éphèse, Démocrite, Diogène Laërce, Héraclite d'Éphèse, Phérécyde de Syros, Platon, Xénophon

\section{AUTEURS}

\section{FABIO STELLA}

it 